\title{
Patientinnen und Patienten in den Mittelpunkt rücken!
}

\section{Christoph Bosshard}

Dr. med., Vizepräsident der FMH, Departementsverantwortlicher DDQ/SAQM

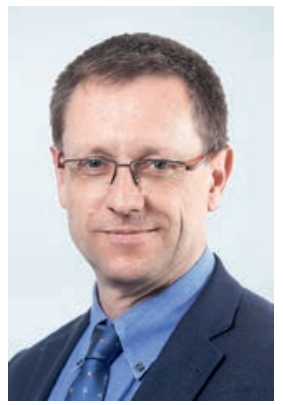

Im klinischen Alltag erleben wir es immer wieder: Wir stehen vor einer Entscheidung, und die Empfehlungen der diversen Richtlinien, die wir im konkreten Kontext berücksichtigen sollten, schliessen sich gegenseitig aus. Nehmen wir als Beispiel einen 70-jährigen Patienten mit frisch erkanntem Vorhofflimmern. Aufgrund des Schlaganfallrisikos ist eine Blutverdünnung angezeigt. Wenn hier nur die stattgehabten Magenblutungen nicht wären ... Guter Rat ist also nicht so banal. Wissenschaftliche Studien und darauf aufbauende Richtlinien haben eben genau dort ihre Grenzen, wo die Studien ihre Ausschlüsse haben. Und gerade die am höchsten bewertete Evidenz der kontrollierten randomisierten Studien zeigt viele solche Ausschlüsse, da sonst die Kollektive nicht ohne zusätzliche Störfaktoren miteinander verglichen werden könnten. Gerade die Multimorbidität ist hier ein stetes Ausschlusskriterium. Letztlich sind auch die Wünsche und Lebensentwürfe unserer Patientinnen und Patienten nicht schematisch einheitlicher Natur. Wir sind nicht nur rein medizinische Individuen! Auch wurde am 2. Dezember 2015 in der Schweizerischen Ärztezeitung mit dem Grundlagenpapier Medizinische Forschung - Wie steht

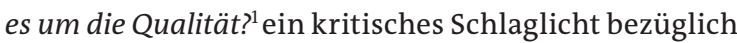
weiterer Störfaktoren publiziert. Somit wird klar: Richtlinien sind wertvolle Hilfsmittel, die uns im Alltag unterstützen können. Sie können uns letztlich jedoch Entscheidungen nicht abnehmen. Also benötigen wir noch ein weiteres komplementäres Element. Ist es nicht naheliegend, dieses Element direkt bei unseren Patientinnen und Patienten zu suchen? Sie sind es nämlich, mit ihrem Leiden, ihren Wünschen, ihren Vorstellungen und Ängsten, welche sich uns anvertrauen! Und genau hier ist der Ansatzpunkt der patientenorientierten Outcome-Erhebung: Was genau führt den Patienten dazu, unseren Rat, unsere Hilfe in Anspruch zu nehmen? Welche Herausforderungen stellen sich in der konkreten Situation, im konkreten Kontext? Hier sind nebst der immer häufiger vorkommenden Multimorbidität auch unterschiedliche individuelle Aspekte ausschlaggebend: Geht es darum, dass die Arbeit wiederaufgenommen werden kann? Geht es um die Reduktion oder Vermeidung einer Pflegebedürftig- keit und somit darum, die Unabhängigkeit eines Patienten zu bewahren? Sollen liebgewonnene Hobbys wieder ermöglicht werden? Überall geht es auch um Selbstwertgefühle und Wertvorstellungen. Und diese sind eben nicht normierbar. Aber sie können, ja sie müssen in den Entscheidungsprozess einfliessen! Wenn solche Vorgehensweisen systematisch unterstützt und mittels geeigneter Hilfsmittel im Alltag umgesetzt werden, so sollen nicht mehr Laborwerte und Röntgenbilder im Mittelpunkt stehen, sondern die reellen Bedürfnisse unserer Patientinnen und Patienten. Idealerweise können diese ohne Zeitdruck bereits im Vorfeld einer Konsultation im Wartezimmer oder von zu Hause aus abgeklärt werden. So kann die wertvolle Zeit in der Sprechstunde vollends dazu genutzt werden, die eruierten Aspekte noch einmal zu besprechen und zu konkretisieren, anstatt die strukturierte Anamneseerhebung dort durchzuführen. In diesem Sinne gilt es, die Systeme so zu bauen, dass eine administrative Entlastung erfolgt und auf keinen Fall eine Mehrbelastung. Wenn schliesslich die Daten in anonymisierter Form vernetzt und aggregiert auswertbar werden, so kann ein Unterstützungssystem gebaut werden, welches Erfahrungswerte vergleichbarer Situationen aus dem klinischen Alltag verfügbar macht. Letztlich wird nur die individuelle Situation entscheiden, ob, wie und in welcher Form ein solcher Prozess Sinn macht. One-fits-for-all-Lösungen werden es auch hier schwer haben. Der Praxisalltag ist nicht mit dem Poliklinikalltag eines Unispitals gleichzusetzen. Ebenfalls sind die Bedürfnisse der einzelnen Fachrichtungen unterschiedlich. Und letztlich sind es unsere Patientinnen und Patienten, welche in ihrer Individualität nicht nur zu respektieren, sondern auch wahrzunehmen sind! Richtlinien einerseits, Erfahrungswerte für die Einzelfälle andererseits sind keine Gegensätze, sondern notwendige komplementäre Ergänzungen, welche uns alle in der individuellen Entscheidungsfindung unterstützen sollen. Den Entscheid werden sie sowohl unseren Patientinnen und Patienten wie auch uns Ärztinnen und Ärzten nicht abnehmen können. Diese Verantwortung müssen wir stets selbst tragen. 